\title{
Descritores quantitativos na determinação da divergência genética entre acessos de tomateiro do grupo cereja
}

\author{
Quantitative descriptors on determining genetic divergence among cherry tomatoes accessions
}

\author{
Mariella Camargo Rocha ${ }^{{ }^{*}}$ Leandro Simões Azeredo Gonçalves ${ }^{\text {II }}$ Fábio Mathias Corrêa ${ }^{\text {III }}$ \\ Rosana RodriguesII Suelem Lima Silva ${ }^{I}$ Antonio Carlos de Souza Abboud \\ Margarida Goréte Ferreira do Carmo ${ }^{\mathrm{I}}$
}

\section{RESUMO}

O cultivo de tomate do grupo cereja vem adquirindo grande importância econômica, sobretudo para os produtores familiares que se utilizam do cultivo orgânico para agregar valor ao seu produto. A introdução de novos genótipos nesse mercado deve ser respaldada por estudos de caracterização e avaliação do germoplasma, os quais, posteriormente, poderão fornecer dados que serão utilizados diretamente pelos produtores ou serão recomendados para programas de melhoramento. O objetivo deste trabalho foi testar 40 genótipos de tomateiro do grupo cereja, de procedencias variadas, sendo 36 acessos da coleção de germoplasma do Departamento de Fitotecnia da UFRRJ e quatro cultivares: 'Joanna', 'Perinha Água Branca (PAB)', 'Samambaia' e o híbrido Super Sweet. As plantas foram cultivadas em condições de campo, sob manejo orgânico, no delineamento de blocos ao acaso com quatro repetições $e$ cinco plantas por parcela no período de junho a dezembro de 2004. As variáveis estudadas foram: produção total de frutos, produção de frutos comerciais e não-comerciais, diâmetro longitudinal e equatorial dos frutos, espessura da polpa, massa mínima, média e máxima de frutos por planta. Os dados foram analisados considerando a distância de Mahalanobis e o agrupamento dos acessos foi realizado com base em UPGMA e pela análise de variáveis canônicas. Para a verificação do ajuste entre a matriz de similaridade e o dendrograma obtido, foi calculado o coeficiente de correlação cofenética (CCC). A importância relativa das características foi determinada pelo método de Singh. Os acessos foram separados em dois grupos, um deles contendo apenas o acesso ENAS 1021, enquanto o outro grupo, com 39 acessos, foi subdividido, gerando a formação de sete subgrupos. O coeficiente de correlação cofenética foi de 0,9, demonstrando que houve um ajuste muito bom entre a matriz de distância e o dendrograma. As três primeiras variáveis canônicas explicaram 78,58\% da variação observada, com a formação de sete grupos. A variável mais importante foi a produção de frutos não-comerciais, que explicou 22,71\% da variação total. A cultivar 'Perinha Água Branca' foi a que produziu menor porcentagem de frutos nãocomerciais $(2,0 \%)$, enquanto o híbrido Super Sweet produziu $8 \%$ de frutos não-comerciais.

Palavras-chave: Solanum lycopersicum, germoplasma, análise multivariada, importância relativa das características.

\begin{abstract}
Cherry tomato is becoming an important crop alternative mainly for small farmers that use family hand labor and use organic management to differentiate their product. The introduction of new genotypes in this market should be supported by germplasm characterization and evaluation. This germplasm could be recommended for farmers or introduced in breeding programs. The aim of this research was to test 40 cherry tomatoes accessions from different sources, being 36 accessions from germplasm collection of Department de Fitotecnia of UFRRJ and four cultivars, namely 'Joanna', 'Perinha Água Branca', 'Samambaia' and Super Sweet. The plants were grown in field conditions, under organic management, in a randomized block design with four replications and five plants per plot, from June to December 2004. The following variables were studied: fruit total yield; commercial and non-commercial fruit yield; longitudinal and equatorial fruit diameter; pulp thickness; minimum, medium and maximum fruit mass variation per plant. The data were analyzed considering Mahalanobis distance and the cluster
\end{abstract}

IDepartamento de Fitotecnia, Fundação Universidade Federal Rural do Rio de Janeiro (UFRRJ), 23890-000, Seropédica, RJ, Brasil. E-mail: marigonnis@ig.com.br.*Autor para correspondência.

ILLaboratório de Melhoramento Genético Vegetal, Fundação Universidade Estadual do Norte Fluminense Darcy Ribeiro (UENF), Campos dos Goytacazes, RJ, Brasil.

IIIDepartamento de Estatística, Fundação Universidade Federal de Lavras (UFLA), Lavras, MG, Brasil. 
analysis was performed using UPGMA (Unweighted Pair-Group Method Using an Arithmetic Average) and canonic variables. The adjustment between the similarity matrix and dendrograma was performed using the cophenetic correlation. The characters relative importance was determined by Singh's method. The accessions were clustered in two groups, one of them with only one accession (ENAS 1021) and the second group, with 39 accessions, was subdivided generating seven subgroups. The cophenetic correlation was 0.9 meaning a very good adjustment between the distance matrix and the dendrogram. The first three canonic variables explained $78.58 \%$ of observed variation, forming seven groups. 'Perinha Agua Branca' was the one that produced lower proportion of non-commercial fruit yield (11.11\%) while Super Sweet produced $48.05 \%$ of damaged fruit.

Key words: Solanum lycopersicum, germplasm, multivariate analysis, relative traits importance

\section{INTRODUÇÃO}

A produção de tomate para o consumo in natura no Brasil sofreu grandes transformações tecnológicas ao longo dos últimos 25 anos, incluindo o melhoramento genético e o desenvolvimento de novas cultivares (SOUZA \& RESENDE, 2003). Houve um crescimento na produção de cerca de $300 \%$, decorrente de um incremento de $50 \%$ na área cultivada e de $150 \%$ na produtividade (IBGE, 2003). Em 2006, a produtividade média brasileira foi de $57.881 \mathrm{~kg} \mathrm{ha}^{-1}$ (IBGE, 2006). A introdução de novos grupos de cultivares, como a do tomate italiano e do cereja, tem aberto perspectivas para a ampliação do mercado de tomate e criado novas demandas de pesquisa visando o desenvolvimento de cultivares destes grupos adaptadas às condições brasileiras. Além da adaptação das cultivares, a sua adequação a condições de cultivo com menor utilização de insumos e defensivos também deve ser objeto de atenção nos trabalhos sobre o tomate.

O tomate do grupo cereja, conhecido pelo mercado consumidor brasileiro desde a década de 90 , é caracterizado, principalmente, por suas propriedades sensoriais, pelo excelente sabor e pela atrativa coloração vermelha e uniforme. Com o crescimento de sua demanda nos mercados dos grandes centros urbanos, é preciso desenvolver sistemas de produção e introduzir novos genótipos, adaptados às condições de cultivo peculiares a este grupo. No Estado do Rio de Janeiro, principalmente, o cultivo deste grupo de tomate está associado a pequenos produtores e à agricultura familiar, sendo que predomina o manejo orgânico como estratégia para buscar uma maior valorização no mercado.

Embora a agricultura orgânica venha se mostrando uma alternativa promissora, principalmente quando atrelada à agricultura familiar, a maioria das pesquisas está ainda voltada para a comparação de produtividade com a agricultura convencional (TRIVELLATO \& FREITAS, 2003). Ensaios visando à caracterização e à avaliação de germoplasmas, incluindo os de tomate para este segmento, ainda são restritos. Igualmente é restrita a avaliação de genótipos de tomate do grupo cereja nas condições brasileiras e pouco se conhece a respeito da variabilidade genética das novas introduções de germoplasma deste grupo. A pesquisa com recursos genéticos é essencial para a conservação da diversidade genética e para o estudo da divergência genética entre acessos, que constitui a base para programas de melhoramento. Ao longo dos anos, houve um aprimoramento das técnicas estatísticas utilizadas para análise de dados referentes à caracterização e à avaliação de germoplasma e, no Brasil, os descritores quantitativos estão entre os mais estudados em hortaliças. $\mathrm{O}$ aumento no uso de técnicas multivariadas para quantificação da divergência genética tem sido verificado já que essas análises permitem considerar simultaneamente inúmeras características (SUDRÉ et al., 2007).

O presente estudo teve como objetivos caracterizar e avaliar acessos de tomateiro do grupo cereja promissores para a produção orgânica, utilizando descritores quantitativos. Além disso, o estudo buscou estimar a divergência genética entre os acessos estudados e determinar a importância relativa das características testadas.

\section{MATERIAL E MÉTODOS}

O experimento foi conduzido no campo no Setor de Horticultura, do Departamento de Fitotecnia, da Universidade Federal Rural do Rio de Janeiro (UFRRJ), localizada em Seropédica, Estado do Rio de Janeiro, no período de junho a dezembro de 2004. Foram avaliados 36 acessos de tomateiro do grupo cereja da coleção de germoplasma do Departamento de Fitotecnia da UFRRJ e quatro cultivares: a cultivar 'Perinha Água Branca'(PAB), proveniente da Feira do Parque da Água Branca, localizada no bairro de Perdizes, no Estado de São Paulo, e plantada na Fazendinha Agroecológica (Sistema Integrado de Produção Agroecológico-SIPA) do convênio Embrapa/Pesagro-Rio/UFRRJ; a cultivar 'Joanna', produzida pelo produtor Everaldo Zonta e cultivada em Itaguaí e região adjacente; a cultivar 'Samambaia', comercializada pela empresa Agristar e o híbrido $\mathrm{F}_{1}$ Super Sweet (Rogers).

Utilizou-se o delineamento de blocos ao acaso com quatro repetições e cinco plantas por parcela. $\mathrm{O}$ ensaio foi conduzido em uma gleba que se 
encontrava em pousio há mais de 10 anos e o solo foi classificado como planossolo com as seguintes características: a) profundidade de 0 a $20 \mathrm{~cm}$ : $\mathrm{pH}_{\text {(água) }}=6,0 ; \mathrm{P}=105 \mathrm{mg} \mathrm{kg}^{-1} ; \mathrm{K}=91 \mathrm{mg} \mathrm{kg}^{-1} ; \mathrm{Ca}=1,9 \mathrm{Cmol}$ $\mathrm{dm}^{-3}$ de TFSA; $\mathrm{Mg}=0,8 \mathrm{Cmol} \mathrm{dm}^{-3} \mathrm{de}$ TFSA; $\mathrm{Al}=0 \mathrm{Cmol}$ $\mathrm{dm}^{-3}$ de TFSA; $\mathrm{H}+\mathrm{Al}=1,8 \mathrm{Cmol}_{c} \mathrm{dm}^{-3}$ de TFSA; $\mathrm{Na}=0,021 \mathrm{Cmol}_{c} \mathrm{dm}^{-3}$ de TFSA; C $=0,93 \%$; e b) profundidade de 20 a $40 \mathrm{~cm}: \mathrm{pH}_{\text {(água) }}=6,1 ; \mathrm{P}=101 \mathrm{mg} \mathrm{kg}^{-1}$; $\mathrm{K}=72 \mathrm{mg} \mathrm{kg}^{-1} ; \mathrm{Ca}=1,8 \mathrm{Cmol}_{\mathrm{c}} \mathrm{dm}^{-3}$ de TFSA; $\mathrm{Mg}=0,9 \mathrm{Cmol} \mathrm{dm}^{-3} \mathrm{de}$ TFSA; $\mathrm{Al}={ }^{\mathrm{C}} \mathrm{Cmol} \mathrm{dm}^{-3} \mathrm{de}$ TFSA; $\mathrm{H}+\mathrm{Al}=1,7 \mathrm{Cmol} \mathrm{dm}^{-3} \mathrm{de}$ TFSA; $\mathrm{Na}=0,013 \mathrm{Cmol}_{\mathrm{c}} \mathrm{dm}^{-3} \mathrm{de}$ TFSA, $\mathrm{C}=0,90 \%$.

Após a aração e a gradagem, foi feito a semeadura da leguminosa crotalária (Crotalaria juncea), para a adubação verde. Aos 50 dias após a semeadura a crotalária foi roçada e incorporada por meio de gradagem. Em seguida, foram realizadas a marcação e a confecção dos canteiros, com 0,40m de altura e sulcamento, em linhas espaçadas a $1,20 \mathrm{~m}$. As covas foram adubadas conforme recomendações de LEAL et al. (2007), com a aplicação de $15 \mathrm{~g} \mathrm{cova}^{-1}$ de termofosfato e $2 \mathrm{~L} \mathrm{cova}^{-1}$ de esterco bovino.

As mudas foram produzidas em bandejas de polipropileno, com 128 células, preenchidas com substrato preparado a partir de terra argilosa adicionada de esterco de curral (3:1 v/v) e mantidas em casa de vegetação fechada e com cobertura de vidro. $\mathrm{Na}$ semeadura foram colocadas duas a três sementes por célula, sendo que em seguida realizado o desbaste, totalizando uma muda por célula. Aos 43 dias após a semeadura, quando todas as mudas apresentavam-se com dois pares de folhas definitivas, foi realizado o transplante para a área experimental, seguido de irrigação.

Após o transplante, foi realizado o manejo orgânico da cultura, com capinas e adição de cobertura morta (palha de grama batatais) a fim de reduzir o crescimento de plantas invasoras e manter a umidade no solo. Aos 28 dias após o transplante (DAT), foi realizado o tutoramento das plantas com auxílio de fitas de plástico, sendo que as plantas foram amarradas em suas bases e foram conduzidas a um fio de arame a dois metros de altura do chão, seguindo as recomendações de LOPES \& STRIPARI (1998). Ao longo do ciclo da cultura, o suprimento das necessidades hídricas foi realizado por meio de um sistema de irrigação localizado (gotejamento), devidamente projetado para a área onde foi conduzido o ensaio. Aos 42DAT, foi realizada uma adubação de cobertura, com a aplicação de $75 \mathrm{~g} \mathrm{cova}^{-1}$ de esterco de galinha e 20 g cova $^{-1}$ de cinza.

Foram realizadas quatro colheitas aos 92, 99, 106 e 112 DAT, quando os frutos estavam nos estádios de maturação verde-maduro e maduro. Durante as quatro colheitas foram realizadas a contagem e a pesagem de frutos e a medição dos diâmetros longitudinal e equatorial dos frutos e da espessura da polpa, com auxílio de um paquímetro manual, e também foram identificados os frutos com sintomas de doenças e anomalias fisiológicas.

As variáveis avaliadas foram produção de total de frutos (kg planta-1) e produção de frutos comerciais e não-comerciais (incidência de defeitos causados por pragas, doenças ou anomalias fisiológicas), diâmetro longitudinal e equatorial dos frutos $(\mathrm{cm})$, espessura da polpa (cm) e peso das massas mínima, média e máxima de frutos por planta (g).

As avaliações realizadas para a definição do número de frutos não-comerciais consideraram a porcentagem de frutos com ocorrência de broca-grande dos frutos (Helicoverpa zea Bod.), broca-pequena dos frutos (Neoleucinodes elegantalis Guenée) e de traçado-tomateiro (Tuta absoluta Meirick), a ocorrência de podridão-mole (Pectobacterium carotovorum subsp. carotovorum) e a antracnose (Colletotrichum spp.) e a ocorrência de frutos com sintomas de rachadura, escaldadura, podridão apical e lóculos abertos, passados ou deformados.

Os dados foram analisados a partir de técnicas multivariadas, utilizando a distância Generalizada de Mahalanobis ( $\left.\mathrm{D}^{2}\right)$. O agrupamento hierárquico foi realizado pelos métodos Unweighted Pair-Group Method Using an Arithmetic Average (UPGMA), Ward e Vizinho Mais Próximo (VMP). O agrupamento foi testado por meio das variáveis canônicas e a importância relativa das características foi calculada pelo método de SINGH (1981). A análise de variáveis canônicas permite avaliar o grau de similaridade genética entre genitores considerando-se a matriz de covariância residual e a matriz de covariância fenotípica entre os caracteres avaliados. As variáveis canônicas, quando utilizadas em estudos de divergência genética, permitem a identificação de indivíduos similares em gráficos bi ou tridimensionais (CRUZ et al., 2004). Para testar a eficiência de cada método de agrupamento hierárquico, foi calculado o coeficiente de correlação cofenética (CCC), pois, quanto maior este valor, maior é a eficiência do método em questão. As análises de distância Generalizada de Mahalanobis e da importância relativa das características foram efetuadas com o programa GENES (CRUZ, 2001), enquanto que para as análises de agrupamento e de correlação cofenética foi utilizado o pacote hcluster, do Programa R (R DEVELOPMENT CORE TEAM, 2006). 


\section{RESULTADOS E DISCUSSÃO}

Houve diferença significativa, pelo teste F, entre os acessos para as características produção total de frutos, produção de frutos não-comerciais, diâmetro longitudinal e equatorial dos frutos, espessura da polpa e para a massa mínima, média e máxima de frutos por planta. O método de agrupamento UPGMA (Figura 1) mostrou-se mais confiável que os métodos Ward e Vizinho Mais Próximo, uma vez que, ao serem comparados os valores de correlação cofenética (CCC) entre os diferentes métodos, o UPGMA obteve um valor de 0,90, enquanto que Ward e VMP tiveram 0,36 e 0,89, respectivamente. Segundo SOKAL \& ROHLF (1962), valores de 0,9 a 1,0 indicam um ajuste muito bom entre a matriz de dissimilaridade e o gráfico (dendrograma).

Um corte realizado a uma distância de 35\% possibilitou a formação de dois grupos, um deles contendo apenas o acesso ENAS 1021 e o segundo grupo reunindo os demais acessos. O acesso ENAS 1021 caracterizou-se por uma menor produção de frutos, tanto em termos totais quanto comerciais. Além disso, esse acesso apresentou diâmetro longitudinal intermediário $(2,6 \mathrm{~cm})$ associado a um dos maiores valores de diâmetro equatorial $(5,3 \mathrm{~cm})$ e espessura de polpa $(0,6 \mathrm{~cm})$. Portanto, os frutos produzidos por este acesso não se enquadram na categoria de tomate do grupo cereja por estarem com diâmetro acima daquele proposto por FERNANDES et al. (2007) para este tipo de fruto.
O segundo grupo, formado por 35 acessos e as quatro cultivares, foram novamente agrupados, resultando em sete subgrupos, quando se considerou um corte em $14 \%$. Com este agrupamento, quatro subgrupos foram formados com apenas um acesso cada (subgrupo I, contendo o acesso ENAS 1009, subgrupo III com o acesso ENAS 1013, subgrupo V com o acesso ENAS 1016 e subgrupo VI com o acesso ENAS 1028). Uma característica marcante comum a todos esses acessos que ficaram isolados em subgrupos distintos foi o diâmetro equatorial acima de $3,5 \mathrm{~cm}$, fazendo com que esses acessos não sejam adequadamente classificados como tomate cereja, segundo a classificação de FERNANDES et al. (2007). Apesar disso, como os frutos são pequenos comparados ao tamanho dos frutos para serem classificados como tomate tipo Santa Cruz, estes acessos poderiam ser comercializados como pertencente a um grupo "cereja especial” ou ainda como “cerejão”. Com base em mais estudos sobre a variabilidade em termos de tamanho e formato de frutos do tipo cereja, uma nova classificação poderá ser proposta. Embora exista para o tomate do segmento "mesa” classificação determinada pelo Ministério da Agricultura, Pecuária e Abastecimento (MAPA), a falta de adoção desta classificação pela cadeia produtiva do tomate é relatada por RODRIGUES et al. (2007). Esses autores afirmam que a existência de mais de uma linguagem de classificação é um dos entraves encontrados no processo produtivo do tomateiro.

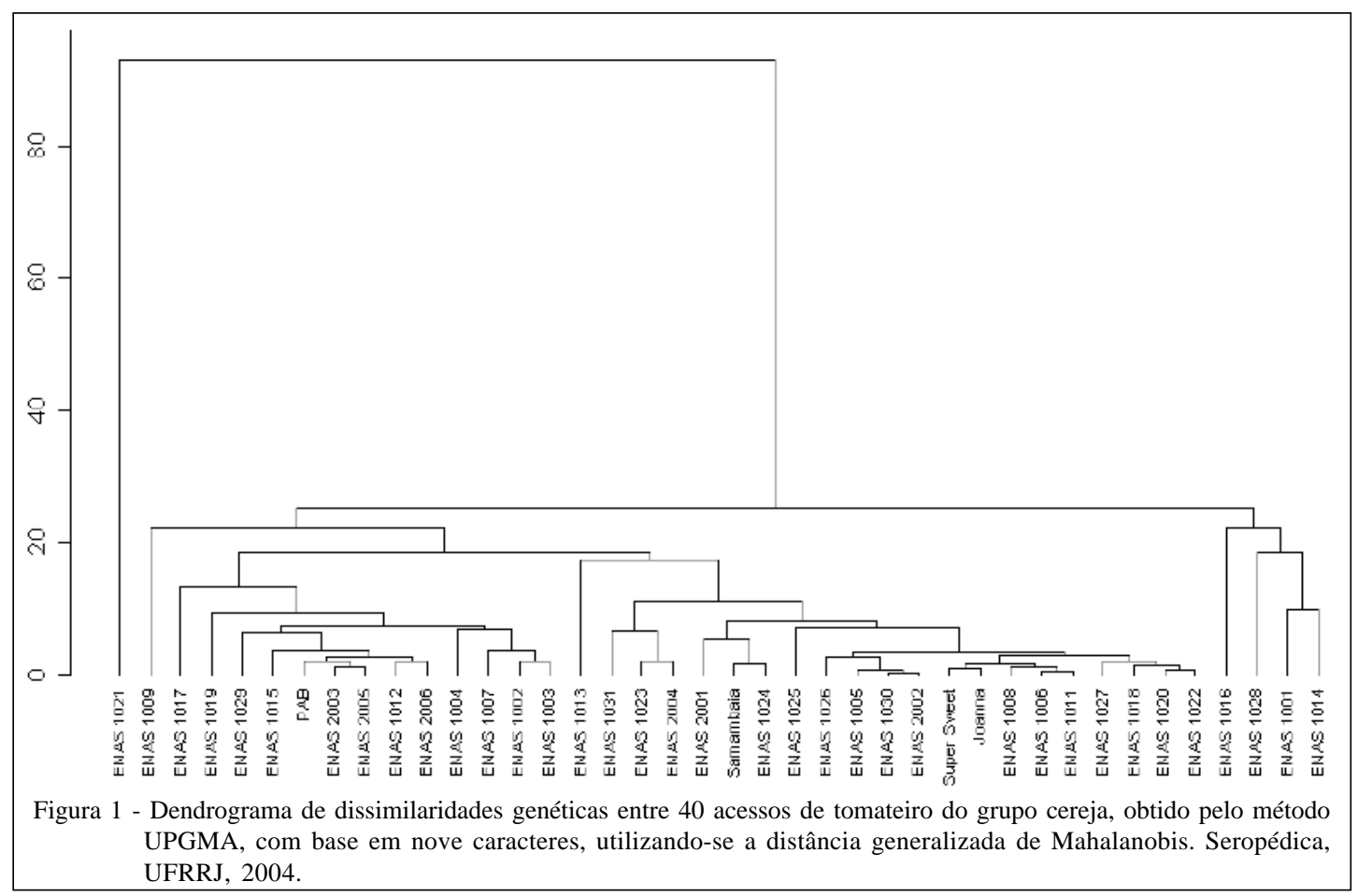

Ciência Rural, v.39, n.3, mai-jun, 2009. 
Os outros subgrupos que reuniram mais de um acesso foram o subgrupo II (13 acessos), o subgrupo IV (20 acessos) e o subgrupo VII (dois acessos). Os acessos do subgrupo II se diferenciaram por produzirem frutos com maior diâmetro longitudinal (variando de 3,4 a 4,5cm) e maior espessura de polpa (valor médio de $0,42 \mathrm{~cm}$ para os acessos deste subgrupo). O subgrupo IV, por sua vez, se diferenciou por produzir frutos com menor diâmetro longitudinal (de 1,9 a $3,7 \mathrm{~cm}$ ) e menor espessura de polpa (valor médio de $0,34 \mathrm{~cm})$.

A formação de dois grupos com base em análise de agrupamento pelo método do Vizinho mais Próximo foi também observada para acessos de tomateiro estudados por KARASAWA et al. (2005). Os autores também optaram pelo subagrupamento dos acessos, uma vez que a estimativa da distância entre pares de indivíduos dentro do grupo foi de elevada magnitude, o que justifica o subagrupamento (ABREU et al., 2004).

Na análise baseada nas variáveis canônicas (VC), foi verificado que cerca de 78,58\% explicaram a variância total, sendo o VC1 responsável por 41,13\%, o VC2 por $27,14 \%$ e o VC3 por 10,31\%, adequando-se, nesse caso, a uma representação gráfica tridimensional (Figura 2). Houve uma concordância parcial entre a análise de agrupamento pelo UPGMA e as variáveis canônicas, quando é observada a distribuição dos acessos em cada grupo, já que alguns acessos foram alocados juntos, independente da técnica de agrupamento utilizada. Os acessos ENAS 1009, ENAS 1013, ENAS 1017, ENAS 1021 e ENAS 1028 formaram diferentes grupos individuais. Com exceção do acesso ENAS 1017, todos os demais também ficaram isolados, compondo um único acesso por grupo ou subgrupo pela análise por UPGMA. O acesso ENAS 1017 produziu menos frutos não-comerciais, com bom diâmetro longitudinal $(4,4 \mathrm{~cm})$. No agrupamento baseado nas VC, a cultivar 'Samambaia' foi alocada junto aos acessos ENAS1001 e ENAS 1014, que, pelo UPGMA, foram agrupados no subgrupo VII e que não corresponderam à classificação de tomate cereja. Observando-se o valor de diâmetro equatorial de 'Samambaia' (3,5cm) concluiu-se que este acesso está no limite estabelecido por FERNANDES et al. (2007). As outras três cultivares testadas se agruparam com outros 26 acessos, enquanto que no UPGMA a cultivar 'Perinha Água Branca' ficou alocada no subgrupo II e as demais cultivares ficaram alocadas no subgrupo IV. Deve-se registrar que, em seleções realizadas por produtores locais, as cultivares 'Perinha Água Branca' e 'Joanna' tiveram o mesmo desempenho agronômico que os genótipos comerciais (Super Sweet e 'Samambaia').

A produção de frutos não-comerciais foi a característica com maior importância relativa, com maior contribuição para a separação dos grupos (Figura 3), com 22,73\%. Os acessos classificados como sendo do

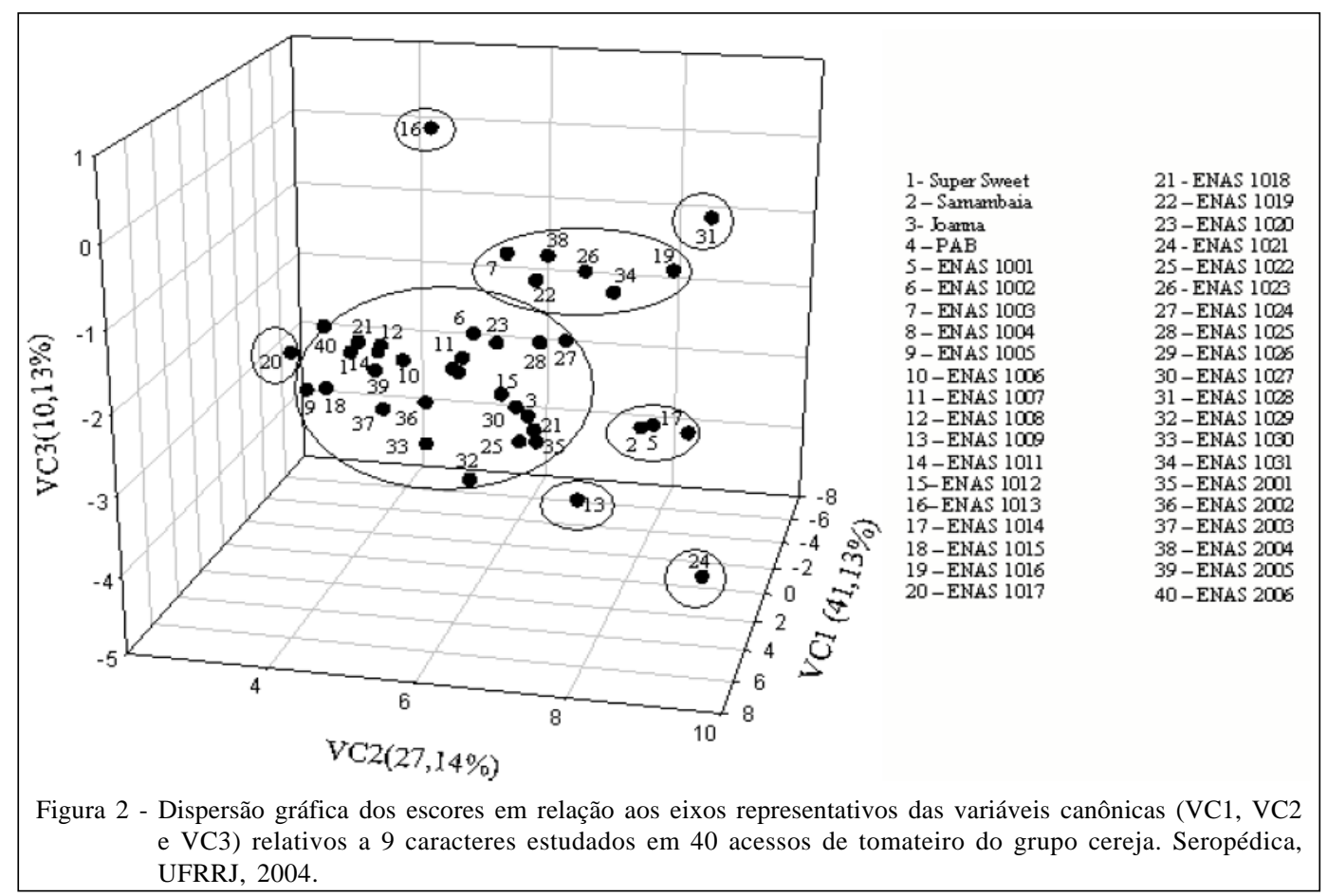

Ciência Rural, v.39, n.3, mai-jun, 2009. 


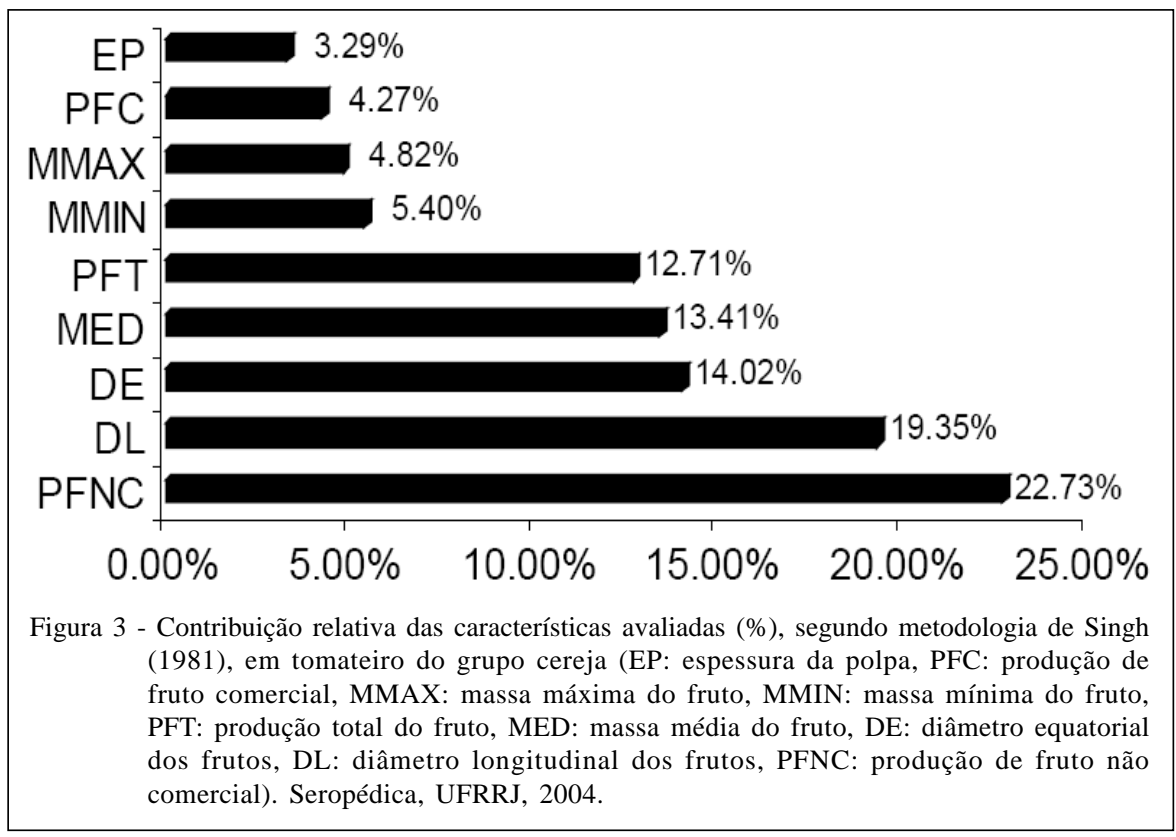

grupo cereja (ENAS 1002, ENAS 1003, ENAS 1019, ENAS 1023, ENAS 1025 e ENAS 1031) tiveram maior produção de frutos não-comerciais e, portanto, não se mostraram promissores, quando consideradas as condições deste experimento. Em relação ao grupo classificado como “cerejão”, destaca-se que ENAS 1009 obteve a menor produção de frutos não-comerciais, portanto, este desempenho deve ser confirmado em outros estudos. A segunda e a terceira característica mais importante foram, respectivamente, os diâmetros longitudinal (19,35\%) e equatorial (14,02\%). Essas características foram marcantes para a discriminação dos grupos, conforme apresentado anteriormente.

Outros trabalhos com hortaliças também mostraram que as características relacionadas à produção, tais como o número de vagens por parcela em plantas de feijão-de-vagem (ABREU et al., 2004) e o número de frutos por planta em Capsicum spp. (BENTO et al., 2007), não foram importantes para a discriminação dos acessos. Entretanto, caracteres relacionados à produção de frutos devem sempre ser avaliados em coleções de germoplasma, pois são úteis na identificação de genótipos superiores para uso em programas de melhoramento.

\section{CONCLUSÕES}

De acordo com os dados referentes ao germoplasma de tomateiro cereja estudado, é possível verificar a existência de variabilidade significativa para os diferentes descritores quantitativos estudados e assim alguns genótipos como ‘Perinha Água Branca’ e 'Joanna', que já são utilizados pelos produtores do Estado do Rio de Janeiro, confirmaram seu potencial de uso em cultivo orgânico. A característica produção de frutos não-comerciais foi a que teve a maior importância relativa para a discriminação dos acessos e a cultivar 'Perinha Água Branca' e outros acessos avaliados tiveram um bom desempenho em relação a este descritor.

\section{AGRADECIMENTOS}

À Coordenação de Aperfeiçoamento de Pessoal de Nível Superior (CAPES), pela concessão da bolsa de Doutorado à primeira autora.

\section{REFERÊNCIAS}

ABREU, F.B. et al. Divergência genética entre acessos de feijãode-vagem de hábito de crescimento indeterminado. Horticultura Brasileira, Brasília, v.22, n.3, p.547-552, 2004.

BENTO, C.S. et al. Descritores qualitativos e multicategóricos na estimativa da variabilidade fenotípica entre acessos de pimentas. Scientia Agraria, Curitiba, v.8, n.2, p.149-156. 2007.

CRUZ, C.D. Programa genes (versão Windows): aplicativo computacional em genética e estatística. Viçosa: UFV, 2001. 648p.

CRUZ, C.D., et al. Modelos biométricos aplicados ao melhoramento de plantas. Viçosa: UFV, 2004. 480p .

FERNANDES, C. et al. Classificação de tomate-cereja em função do tamanho e peso dos frutos. Horticultura Brasileira, Brasília, v.25, n.2, p.275-278. 2007. 
IBGE Brasil, 2003. Acessado em 15 set. 2007. On line. Disponivel em: www.sidra.ibge.gov.br.

IBGE Brasil, 2006. Acessado em 23 mar. 2008. On line. Disponivel em: www.sidra.ibge.gov.br.

KARASAWA, M. et al. Aplicação de métodos de agrupamento na quantificação da divergência genética entre acessos de tomateiro. Horticultura Brasileira, Brasília, v.23, n.4, p.1000-1005, 2005.

LEAL, M.A.A. et al. Utilização de compostos orgânicos como substratos na produção de mudas de hortaliças. Horticultura Brasileira, Brasília, v.25, n.4, p.392-395. 2007.

LOPES, M.C.; STRIPARI, P.C. A cultura do tomateiro. In: GOTO, R; TIVELli, S.W. Produção de hortaliças em ambiente protegido: condições subtropicais. São Paulo: UNESP, 1998. Cap.9, p.257-304.

R DEVELOPMENT CORE TEAM. A language and environment for statistical computing. Vienna: $R$ Foundation for Statistical Computing, 2006. Capturado em 13 de mar. de 2008. Online. Disponível na Internet: http://www.rproject.org
RODRIGUES, L.R. et al. Classificação do tomate por atacadistas e produtores Curitiba. Horticultura Brasileira, Brasília, v.25, n.4, p.521-526, 2007.

SINGH, D. The relative importance of characters affecting genetic divergence. Indian Journal of Genetic and Plant Breeding, New Delhi, v.41, n.2, p.237-245, 1981.

SOKAL, R.R.; ROHLF, F.J. The comparison of dendrograms by objective methods. Taxon, Utrecht, v.11, p.33-40, 1962.

SOUZA, J.L.; RESENDE, P. Manual de horticultura orgânica. Viçosa: Aprenda Fácil, 2003. 564p.

SUDRÉ, C.P. et al. Genetic resources of vegetable crops: a survey in the Brazilian germplasm collections pictured through papers published in the journals of the Brazilian Society for Horticultural Science. Horticultura Brasileira, Brasília, v.25, n.4, p.496-503, 2007.

TRIVELLATO, M.D.; FREITAS, G.B. Panorama da agricultura orgânica. In: STRINGUETA, P.C; MUNIZ, J.N. Alimentos orgânicos: produção tecnologia e certificação. Viçosa: UFV, 2003. p.9-35. 